\title{
Moving homology classes in finite covers of graphs
}

\author{
Benson Farb and Sebastian Hensel *
}

July 29, 2021

\begin{abstract}
Let $Y \rightarrow X$ be a finite normal cover of a wedge of $n \geq 3$ circles. We prove that for any $v \neq 0 \in H_{1}(Y ; \mathbb{Q})$ there exists a lift $\widetilde{F}$ to $Y$ of a homotopy equivalence $F: X \rightarrow X$ so that the set of iterates $\left\{\widetilde{F}^{d}(v): d \in \mathbb{Z}\right\} \subseteq H_{1}(Y ; \mathbb{Q})$ is infinite. The main achievement of this paper is the use of representation theory to prove the existence of a purely topological object that seems to be inaccessible via topology.
\end{abstract}

\section{Introduction}

The group $\operatorname{Out}\left(F_{n}\right)$ of outer automorphisms of the free group $F_{n}$ is isomorphic to the group of homotopy classes of homotopy equivalences of a wedge $X$ of $n$ circles. For any finite regular cover $f: Y \rightarrow X$ with covering group $G$, consider the finite-index subgroup $\Gamma_{G}<\operatorname{Out}\left(F_{n}\right)$ of those homotopy equivalences which lift to $Y$ and act trivially on $G$. There is a representation $\Gamma_{G} \rightarrow \mathrm{GL}\left(H_{1}(Y ; \mathbb{Q})\right)$. Analogous representations of mapping class groups are known as higher Prym representations (see [L, $\mathrm{PW}]$ ).

As one varies $G$, this gives a rich and useful family of representations of $\Gamma_{G}$. These have been studied by Grunewald-Lubotzky and others; see [GL] and the references contained therein. A basic topological problem that arises from this situation is: given an arbitrary finite normal cover $Y \rightarrow X$ with covering group $G$, must it be true that the $\Gamma_{G}$-orbit of any vector $v$ in $H_{1}(Y ; \mathbb{Q})$ is infinite? The corresponding problem with $X$ replaced by a surface and $\operatorname{Out}\left(F_{n}\right)$ replaced by the mapping class group was posed by Putman-Wieland [PW]. They proved that this problem is essentially equivalent to the vanishing of the virtual first Betti number of the mapping class group, a major open problem posed by Ivanov; see [PW] for a detailed discussion.

For very special $G$-covers $Y \rightarrow X$, the representation of $\Gamma_{G}<\operatorname{Out}\left(F_{n}\right)$ on $H_{1}(Y ; \mathbb{Q})$ can be worked out explicitly, giving a positive answer to this question; see [GL, GLLM], and [L, McM] for related work on finite abelian covers of surfaces. For arbitrary $G$ it is not feasible to identify the actual representation of $\Gamma_{G}$ explicitly. Instead, one must construct individual automorphisms of $F_{n}$ to move a given vector in $H_{1}(Y ; \mathbb{Q})$.

One of the aspects that makes the Putman-Wieland problem challenging is that the elements of $\Gamma_{G}$ act on $Y$ in a highly constrained way: each must intertwine the $G$-action. However, for general covers it is far from clear (and indeed probably false in general) that

\footnotetext{
*The first author gratefully acknowledges support from the National Science Foundation.
} 
every automorphism of $H_{1}(Y ; \mathbb{Q})$ which homologically intertwines correctly with $G$ actually arises as a lift! This makes it difficult to understand and control $\Gamma_{G}$-orbits of vectors.

In this paper we solve the Putnam-Wieland problem for graphs.

Theorem 1.1 (Moving homology classes in covers). Let $f: Y \rightarrow X$ be a finite $G$-cover with $\operatorname{rank}\left(\pi_{1}(X)\right) \geq 3$. Then the $\Gamma_{G}$-orbit of any $0 \neq v \in H_{1}(Y ; \mathbb{Q})$ is infinite. In fact, given any $0 \neq v \in H_{1}(Y ; \mathbb{Q})$, there exists a homotopy equivalence $F: X \rightarrow X$ such that $F$ lifts to $\widetilde{F}: Y \rightarrow Y$ and so that the set $\left\{\widetilde{F}^{d}(v): d \in \mathbb{Z}\right\} \subseteq H_{1}(Y ; \mathbb{Q})$ is infinite.

The statement of Theorem 1.1 is false if $\operatorname{rank}\left(\pi_{1}(X)\right)=2$ : we prove in $\S 3.1$ that the commutator of a pair of generators of $\pi_{1}(X)$ is fixed under all of $\operatorname{Out}\left(F_{2}\right)$, and we use this to constuct a homology class with finite orbit. In a similar vein, we want to mention that Carlos Matheus [M] has observed that there is an example of a cover of a genus 2 surface in which the Putman-Wieland problem is false.

The key idea of the proof of Theorem 1.1. To prove Theorem 1.1 we must, given an arbitrary $v \neq 0 \in H_{1}(Y ; \mathbb{Q})$, construct out of nowhere a homotopy equivalence $F: X \rightarrow X$ with the desired properties. We first reduce this problem to the problem of finding a loop $\ell$ in $X$ satisfying some very special properties; we then show that an "edge-slide" along $\ell$ is the required $F$. Even in simple examples the loop $\ell$ that actually works is extremely complicated (see $\S 3.2$, so how can we find it in general? The key is to use the structure of $H_{1}(Y ; \mathbb{Q})$ as a $G$-representation. This representation was computed in 1934 by Chevalley-Weil [CW] for surfaces, and later by Gaschütz for graphs (see [GLLM]).

Theorem 1.2 (Chevalley-Weil for graphs [CW, GLLM]). Let $X$ be a finite graph with $\pi_{1}(X)$ free of rank $n \geq 2$. Let $Y \rightarrow X$ be a finite cover with deck group $G$. Then as $G$-representations:

$$
H_{1}(Y ; \mathbb{Q}) \cong \mathbb{Q}[G]^{n-1} \oplus \mathbb{Q}
$$

where $\mathbb{Q}[G]$ denotes the regular representation and $\mathbb{Q}$ the trivial representation of $G$.

Using this information about the $G$-representation $H_{1}(Y ; \mathbb{Q})$ we construct an $\ell$ so that the corresponding edge-slide map has the desired properties. The novelty here is that we have constructed a topological object non-explicitly, via representation theory, that seems inaccessible via purely topological methods. For example, in most covers the loop $\ell$ cannot be represented by a simple closed curve in any identification of the free group $F_{n}$ with the fundamental group of a punctured surface (see $\S 3.2$. .

\section{Proof of the main theorem}

In this section we prove Theorem 1.1 in a sequence of steps. We begin by setting up some notational conventions that are used throughout the article.

Fix $n \geq 3$ and let $X$ be the wedge of $n$ copies of $S^{1}$. Let $p$ be the point at which the circles are joined. We pick once and for all an orientation on each copy of $S^{1}$, and we label these oriented edges of $X$ by $a_{1}, \ldots, a_{n}$. We identify $a_{i}$ with the corresponding generator of $\pi_{1}(X, p)=F_{n}$. Let $f: Y \rightarrow X$ be any finite normal cover of $X$ and let $G=\pi_{1}(X) / \pi_{1}(Y)$ be the corresponding deck group. We denote the quotient homomorphism by $q: F_{n} \rightarrow G$. 
The vertices of the graph $Y$ are exactly the elements in the preimage $f^{-1}(p)$ of the (unique) vertex of $X$. Each edges of $Y$ maps by $f$ to a petal $a_{i}$ of $X$ injectively except at the endpoints. Choose a preferred basepoint $\hat{p} \in f^{-1}(p)$, which we denote as the identity vertex. We label each other vertex $\hat{p}^{\prime} \in f^{-1}(p)$ by the element $g \in G$ such that $g \hat{p}=\hat{p}^{\prime}$. Each vertex $\hat{p}^{\prime}$ of $Y$ is of valence $2 n$ since a small neighborhood of the basepoint $p$ in $X$ lifts homeomorphically to $Y$. We label each half-edge adjacent to $\hat{p}^{\prime}$ with $a_{i}$ or $a_{i}^{-1}$, depending on which half-edge of $X$ it lifts (interpreted as maps $[0,1] \rightarrow X$ ). Observing that the action of the deck group is the action obtained by path-lifting yields the following

Observation 2.1. $Y$ is the Cayley graph of the group $G$ with respect to the generating set $q\left(a_{1}\right), \ldots, q\left(a_{n}\right)$.

Let us emphasize that the generating set $q\left(a_{1}\right), \ldots, q\left(a_{n}\right)$ is not symmetric (and may contain identity elements) and thus the graph $Y$ only has edges corresponding to right multiplication of the $q\left(a_{i}\right)$ (and may have loops).

We are now ready to begin the proof in earnest.

Step 1 (A cohomology class that pairs with $v$ ): Fix any edge $e \in Y$. Let $\xi_{e}$ be the cocycle defined as follows. Given an arbitrary 1-cycle $\sigma$, write it as a weighted sum of edges $\sigma=c e+\sum_{e^{\prime} \neq e} c_{e^{\prime}} e^{\prime} \in C_{1}(Y)$ and define $\xi_{e}(\sigma):=c$. The set $\left\{\xi_{e}: e\right.$ is an edge of $\left.Y\right\}$ spans $H^{1}(Y ; \mathbb{Q})$. Since each edge of $Y$ is the lift of some $a_{i}$, it follows that we can relabel the $a_{i}$ so that there exists a lift $\alpha$ of $a_{1}$ to $Y$ so that $\xi_{\alpha}(v) \neq 0$.

Step 2 (Edge sliding maps and the action of their lifts): Let $\ell \subset X$ be any loop that intersects $a_{1}$ only in the basepoint $x_{0} \in X$. We define a homotopy equivalence $\mathrm{sl}_{\ell}: X \rightarrow X$ by taking the initial point of $a_{1}$ and dragging it along $\ell$. On the level of $\pi_{1}(X)$, we have that $\left(\mathrm{sl}_{\ell}\right)_{*}$ maps $a_{1}$ to $\ell * a_{1}$ and fixes all other generators $a_{2}, \ldots, a_{n}$ of $\pi_{1}(X)$. Since $X$ is an Eilenberg-MacLane space, the automorphism $\mathrm{sl}_{*}$ determines the homotopy equivalence $\mathrm{sl}_{\ell}$ up to homotopy.

Lemma 2.2 (Action of a lifted edge-slide on $\boldsymbol{H}_{\mathbf{1}}$ ). Suppose $\ell$ is a based loop in $X$. If $\ell$ lifts to a closed loop $\tilde{\ell}$ based at the identity vertex in $Y$ then $\mathrm{sl}_{\ell}$ lifts to a homotopy equivalence $F: Y \rightarrow Y$ such that $F_{*}: H_{1}(Y ; \mathbb{Q}) \rightarrow H_{1}(Y ; \mathbb{Q})$ acts as

$$
F_{*}(w)=w+\sum_{g \in G} \xi_{g \cdot \alpha}(w)[g \cdot \tilde{\ell}]
$$

where $[g \cdot \tilde{\ell}]$ denotes the class of $g \cdot \tilde{\ell}$ in $H_{1}(Y ; \mathbb{Q})$.

Proof. The homotopy equivalence $\mathrm{sl}_{\ell}$ fixes the basepoint of $X$. Recall that we have a surjection $q: F_{n} \rightarrow G$ defining the cover $Y$. Since by assumption $\ell$ lifts to a closed loop in $Y$, we have $q(\ell)=0$. Therefore $\left(\mathrm{sl}_{\ell}\right)_{*}: F_{n} \rightarrow F_{n}$ preserves the kernel $\operatorname{ker}(q)$. As a consequence, $\mathrm{sl}_{\ell}$ lifts to $Y$.

Let $F$ denote the lift of $\operatorname{sl}_{\ell}$ that fixes the identity vertex of $Y$. Since $q(\ell)=0$ it follows that $\left(\mathrm{sl}_{\ell}\right)_{*}$ induces the identity automorphism $G \rightarrow G$. Thus, for every element $g \in G$ in the deck group,

$$
F(g \cdot x)=\mathrm{sl}_{*}(g) \cdot F(x)=g \cdot F(x)
$$


In particular, $F$ fixes each vertex of $Y$ because $X$ has a single vertex and therefore each vertex of $Y$ is the image of the identity vertex under some $g \in G$.

Let $Y_{0}$ denote the connected component of $Y-\operatorname{interior}\left(p^{-1}\left(a_{1}\right)\right)$ containing the identity vertex. The restriction $f: Y_{0} \rightarrow X-a_{1}$ is a covering map, and $\mathrm{sl}_{\ell}$ restricts to the identity map on $X-a_{1}$. Thus $\left.F\right|_{Y_{0}}$ is a lift of the identity that fixes the identity vertex $\widetilde{b}$ in $Y$, and so $F$ restricts to the identity on $Y_{0}$. By Equation (3) $F$ restricts to the identity on each component $Y_{i}$ of $Y-p^{-1}\left(a_{1}\right)$ because each $Y_{i}$ is the $g$-translate of $Y_{0}$ for some $g \in G$. This implies that each edge of $Y$ labeled by any $a_{i} \neq a_{1}$ is also fixed by $F$, since any such edge is contained in some $Y_{i}$.

Now consider the (unique) oriented edge $\widetilde{a}_{1}$ based at $\widetilde{b}$ which is a lift of $a_{1}$. The definition of lifting a map via lifting paths shows that $F$ maps $\widetilde{a}_{1}$ to the edge-path $\widetilde{\ell} * \widetilde{a}_{1}$. Equation $(3)$ implies that $F$ maps $g \cdot \widetilde{a}_{1}$ to the edge-path $(g \cdot \widetilde{\ell}) *\left(g \cdot \widetilde{a}_{1}\right)$.

Since $F$ fixes each vertex in $Y$ and maps each edge to an edge-path, $F$ induces a chain map $F_{\#}$ on the chain complex $C_{*}(Y)$. The discussion above implies that, for each oriented edge $\gamma$ of $Y$,

$$
F_{\#}(\gamma)= \begin{cases}\gamma & \text { if } \gamma \text { is labeled by } a_{j}, j>1 \\ \gamma+g \cdot \tilde{\ell} & \text { if } \gamma=g \cdot \widetilde{a}_{1}\end{cases}
$$

Thus for each oriented edge $\gamma$ :

$$
F_{\#}(\gamma)=\gamma+\sum_{g \in G} \xi_{g \cdot \alpha}(\gamma)[g \cdot \tilde{\ell}]
$$

because $\xi_{g \cdot \alpha}(\gamma)=1$ if $\gamma=g \cdot \alpha$ and $\xi_{g \cdot \alpha}(\gamma)=0$ otherwise.

Because the oriented edges of $Y$ form a basis of $C_{1}(Y)$ and $F_{\#}$ is a chain map, Equation (4) holds for all $\gamma \in C_{1}(Y)$. Taking the induced map $F_{*}$ on $H_{1}(Y ; \mathbb{Q})$ gives Equation $(2)$, as desired.

Step 3 (The right choice of $\ell$ implies the theorem): Suppose that we can find a based loop $\ell \subset X$ satisfying each of the following:

1. $\ell$ does not intersect the interior of the edge $a_{1}$.

2. $\ell$ lifts to a closed loop $\tilde{\ell}$ based at the identity vertex in $Y$.

3. The set $\{G \cdot[\tilde{\ell}]\} \subset H_{1}(Y)$ is linearly independent.

Under these conditions, $\mathrm{sl}_{\ell}$ lifts to a homotopy equivalence $F: Y \rightarrow Y$, and we will prove below that

$$
F^{n}(w)=w+\sum_{g \in G} n \xi_{g \cdot \alpha}(w)[g \cdot \tilde{\ell}]
$$

for all $n>0$

Since $\xi_{\alpha}(v) \neq 0$, Equation (5) with $w=v$ implies that the coefficient of [ $\left.\ell\right]$ in $F^{n}(v)$ is unbounded as $n$ increases. The assumption that $\{G \cdot[\tilde{\ell}]\} \subset H_{1}(Y)$ is linearly independent gives that all of the $F^{n}(v)$ are distinct, thus proving the theorem.

To prove that Equation (5) holds, first note that $\xi_{g \cdot \alpha}\left(g^{\prime} \cdot \widetilde{\ell}\right)=0$ for all $g, g^{\prime} \in G$ since no lift of $\ell$ intersects any lift of the edge $a_{1}$. Thus $\xi_{g \cdot \alpha}(F(w))=\xi_{g \cdot \alpha}(w)$ by Equation (2). 
Equation (2) and induction on $n$ now give:

$$
\begin{aligned}
F^{n+1}(w)=F\left(F^{n}(w)\right) & =F^{n}(w)+\sum_{g \in G} \xi_{g \cdot \alpha}\left(F^{n}(w)\right)[g \cdot \tilde{\ell}] \\
& =F^{n}(w)+\sum_{g \in G} \xi_{g \cdot \alpha}(w)[g \cdot \tilde{\ell}] \\
& =w+\sum_{g \in G} n \xi_{g \cdot \alpha}(w)[g \cdot \tilde{\ell}]+\sum_{g \in G} \xi_{g \cdot \alpha}(w)[g \cdot \tilde{\ell}] \\
& =w+\sum_{g \in G}(n+1) \xi_{g \cdot \alpha}(w)[g \cdot \tilde{\ell}]
\end{aligned}
$$

and so Equation (5) follows by induction.

Step 4 (Finding the $\ell$ ): By Step 3, to prove the theorem it is enough to find a based loop $\ell \subset X$ satisfying the three assumptions 1-3 stated in Step 3. We do this via representation theory.

Recall that we have a surjection $q: F_{n} \rightarrow G$. Let

$$
G_{0}:=q\left(\left\langle a_{2}, \ldots, a_{n}\right\rangle\right)
$$

Let $Y_{0}$ denote the connected component of $Y$ - interior $\left(p^{-1}\left(a_{1}\right)\right)$ containing the identity vertex. The restriction of the covering map $f: Y \rightarrow X$ to $Y_{0}$ is a regular cover with deck group $G_{0}$. Thus, by Theorem 1.2 , we have the following.

Observation 2.3. $H_{1}\left(Y_{0} ; \mathbb{Q}\right)$ is isomorphic as a $G_{0}$-module to $\mathbb{Q}\left[G_{0}\right]^{n-2} \oplus \mathbb{Q}$.

Now note that $Y$ - interior $\left(p^{-1}\left(a_{1}\right)\right)$ is the union of the translates $Y_{0}, Y_{1}, \ldots, Y_{k}$ of the subgraph $Y_{0}$ under the deck group $G$; here $k=\left[G: G_{0}\right]$. The reason for this is that $p^{-1}\left(a_{1}\right)$ is $G$-invariant, and hence so is its complement.

Claim 2.4. The map

$$
I: \oplus_{j=0}^{k} H_{1}\left(Y_{j}\right) \rightarrow H_{1}(Y)
$$

induced by the inclusions $Y_{j} \rightarrow Y$ is injective.

Proof. First note that the graphs $Y_{j}$ are disjoint, and each vertex of $Y$ is contained in some $Y_{j}$.

By collapsing edges of $Y$ with distinct endpoints, we can ensure that each $Y_{j}$ is a rose (i.e. has a single vertex). Since such collapses are homotopy equivalences, this modification does not change injectivity of the map $I$. Similarly, we can collapse further edges in the complement of the $Y_{j}$ to ensure that $Y$ becomes itself a rose, again without changing $I$. In the resulting rose, the claim is clear since each $Y_{i}$ is a "sub-rose", and any two of these sub-roses intersect only at the one remaining vertex.

Now choose any $u \in H_{1}\left(Y_{0} ; \mathbb{Q}\right)$ so that $\left\{G_{0} \cdot u\right\}$ is linearly independent; this is possible by considering $u \in \mathbb{Z}\left[G_{0}\right] \subset \mathbb{Q}\left[G_{0}\right]^{n-2} \oplus \mathbb{Q} \cong H_{1}\left(Y_{0} ; \mathbb{Q}\right)$ of the form $u=1 \cdot g_{0}$ for any $g_{0} \in G_{0}$.

Claim 2.5. The set $\{g \cdot u: g \in G\} \subset H_{1}(Y ; \mathbb{Q})$ is linearly independent. 
Proof. Suppose that

$$
\sum_{g \in G} c_{g} g u=0
$$

We rewrite this as a sum over coset representatives,

$$
\sum_{h \in G / G_{0}} \sum_{g_{0} \in G_{0}} c_{h g_{0}} h g_{0} u=0
$$

By Claim 2.4 we then see that for each $h \in G / G_{0}$ we have

$$
0=\sum_{g_{0} \in G} c_{h g_{0}} h g_{0} u=h\left(\sum_{g_{0} \in G} c_{h g_{0}} g_{0} u\right)
$$

and thus

$$
0=\sum_{g_{0} \in G} c_{h g_{0}} g_{0} u
$$

By the defining property of $u$ this however implies $c_{h g_{0}}=0$ for all $g_{0} \in G_{0}$. Since this is true for all $h, g_{0}$ we see that $c_{g}=0$ for all $g \in G$.

Hence, any loop $\ell \subset Y_{0}$ defining the element $u \in H_{1}\left(Y_{0}\right)$ has the desired properties 1-3. This finishes the proof of Theorem 1.1 .

\section{Complexity of $\ell$}

Even in the simplest cases, the simplest loop $\ell$ with the required properties 1-3 is necessarily complicated from a topological perspective (and the complexity grows with the degree of the cover). By using representation theory, we were able to completely bypass this issue, but in this section we collect two observations which indicate the complexity of the desired element.

\section{1 $\ell$ is complicated as a word}

In this section we describe explicitly a simple but nontrivial example of the homology of a cover as a representation. The purpose of this example is twofold - on the one hand it will show that even in the simplest cases, words $\ell$ as required in the proof of Theorem 1.1 are fairly complicated. As a byproduct, we will show that Theorem 1.1 is false in rank 2 .

Let $X$ be the wedge of two circles. To simplify notation, denote the generators of $\pi_{1}(X)$ by $a, b$. Let $Y$ be the cover of $X$ corresponding to the kernel of the map $q: \pi_{1}(X) \rightarrow H_{1}(X ; \mathbb{Z} / 2 \mathbb{Z})$. This is a 4 -fold cover with deck group $\mathbb{Z} / 2 \mathbb{Z} \times \mathbb{Z} / 2 \mathbb{Z}$. Theorem 1.2 implies that

$$
H_{1}(Y ; \mathbb{Q})=\mathbb{Q}^{2} \oplus V_{a} \oplus V_{b} \oplus V_{a b}
$$

where $V_{a}, V_{b}, V_{a b}$ are the three nontrivial rational irreducible representations of $G=\mathbb{Z} / 2 \mathbb{Z} \times$ $\mathbb{Z} / 2 \mathbb{Z}$. Each of these representations is 1-dimensional, and the representations are given as follows:

$$
\left.q(a)\right|_{V_{a}}(z)=z,\left.\quad q(b)\right|_{V_{a}}(z)=-z
$$




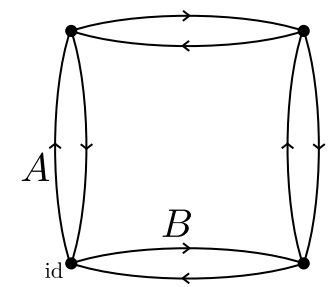

Figure 1: The mod-2 homology cover

$$
\begin{gathered}
\left.q(a)\right|_{V_{b}}(z)=-z,\left.\quad q(b)\right|_{V_{b}}(z)=z \\
\left.q(a)\right|_{V_{a b}}(z)=-z,\left.\quad q(b)\right|_{V_{a b}}(z)=-z
\end{gathered}
$$

In order to find these representations topologically, we begin by considering the lifts $A, B$ of $a^{2}, b^{2}$ respectively to the identity basepoint. We then see the following

$$
\begin{aligned}
V_{a} & =\langle A-q(b) A\rangle \\
V_{b} & =\langle B-q(a) B\rangle
\end{aligned}
$$

which follows simply as the representation is the correct one. To describe $V_{a b}$, let $C$ be the elevation of $a b$ at the identity vertex and let $C^{\prime}$ be the elevation of $b a$ at the identity vertex. It is easy to check that

$$
V_{a b}=\left\langle C-C^{\prime}\right\rangle .
$$

Note that each of these three irreducible representations can be generated by linear combinations of elevations of primitive elements - but not by elevations of primitive elements themselves. This is indicative of the situation for general covers.

The trivial representation is the image of the transfer map:

$$
\mathbb{Q}^{2}=\langle A+q(b) A, B+q(a) B\rangle
$$

As a final note, we will describe an element $x \in H_{1}(Y ; \mathbb{Q})$ so that $\langle G x\rangle \simeq \mathbb{Q}[G]$ (such an element is not unique). Such an $x$ necessarily needs to contain a contribution from each irreducible representation; here we can simply add the bases of $V_{a}, V_{b}, V_{a b}$ and something nonzero in the transfer part. One example is the lift of the element

$$
a^{3} b^{-1} a b \text {. }
$$

Note that, again, this element is not primitive, but $x$ is a linear combination of elevations of primitive elements. In fact, elements with the defining property of $x$ usually cannot be equal to elevations of primitive elements (as will be shown in the next section).

Also note that for more complicated $G$, the complexity of $\ell$ will increase further, as more irreducible representations appear in $\mathbb{Q}[G]$.

Finally, note that the loop representing the commutator $[a, b]$ lifts in $Y$ to a homologically nontrivial loop. The group $\operatorname{Out}\left(F_{2}\right)$ fixes the conjugacy class defined by $[a, b]$, and hence any lift of an element in $\operatorname{Out}\left(F_{2}\right)$ permutes the set of lifts of $[a, b]$. This shows

Lemma 3.1. There are covers $Y \rightarrow X$ of a rank 2 graph $X$, and homology classes $v \in$ $H_{1}(Y ; \mathbb{Q})$ so that the set

$$
\left\{\widetilde{\phi}_{*}(v) \mid \phi \in \operatorname{Out}\left(F_{n}\right) \text { lifts to } Y\right\}
$$

is finite. 


\section{$3.2 \ell$ is complicated as a topological object}

Here, we note the following simple observation

Lemma 3.2. Let $Y \rightarrow X$ be a regular cover of graphs with the property that no primitive element in $\pi_{1}(X)$ lifts (with degree 1 ) to $Y$. Then any element $\ell$ as in the proof of Theorem 1.1 cannot be represented by a simple closed curve with respect to any identification $\pi_{1}(X) \cong \pi_{1}(\Sigma)$ for a punctured surface $\Sigma$. More generally, any element $\ell$ satisfying only property 3 cannot map in $\pi_{1}(X)$ to a multiple of a simple closed curve.

Proof. Let the identification $\pi_{1}(X) \cong \pi_{1}(\Sigma)$ be given, and let $\Sigma^{\prime} \rightarrow \Sigma$ be the cover of $\Sigma$ defined by $\pi_{1}(Y)$. Note that any element given by a simple closed curve in $\pi_{1}(\Sigma)$ is primitive. Hence, given any simple closed curve $\gamma$, the preimage of $\gamma$ in $\Sigma^{\prime}$ consists of strictly less than $|G|$ elements; hence the span of these elements is of rank strictly less than $|G|$. Thus, condition 3. of the defining properties of $\ell$ cannot hold.

We remark that covers as in the lemma are plentiful; in particular any cover that covers the mod- $n$ homology cover of $X$ has the desired property.

\section{References}

[CW] C. Chevalley and A. Weil, Über das Verhalten der Integrale 1. Gattung bei Automorphismen des Funktionenkörpers. Abh. Math. Sem. Univ. Hamburg, 10:358361, 1934.

[FM] B. Farb and D. Margalit, A primer on mapping class groups, to appear in Princeton Mathematical Series, Princeton University Press. Available at: http://www.math.utah.edu/ margalit/primer/

[GLLM] F. Grunewald, M. Larsen, A. Lubotzky and J. Malestein, Arithmetic quotients of the mapping class group, preprint, April, 2015.

[GL] F. Grunewald and A. Lubotzky, Linear representations of the automorphism group of a free group, Geom. Funct. Anal. 18 (2009), no. 5, 15641608.

[L] E. Looijenga, Prym Representations of Mapping Class Groups, Geometriae Dedicata 64 (1997), 69-83.

[M] C. Matheus, Some comments on the conjectures of Ivanov and Putman-Wieland, https://matheuscmss.wordpress.com/2015/04/24/some-comments-on-the-conjecturesof-ivanov-and-putman-wieland/

[McM] C. T. McMullen, Braid groups and Hodge theory, Mathematische Annalen 355, no. 3 (2013), 893-946.

[PW] A. Putman and B. Wieland, Abelian quotients of subgroups of the mappings class group and higher Prym representations, J. Lond. Math. Soc. (2) 88 (2013), no. 1, 79-96.

Benson Farb

Department of Mathematics

University of Chicago

5734 University Ave.

Chicago, IL 60637

E-mail: farb@math.uchicago.edu
Sebastian Hensel

Mathematisches Institut

Rheinische Friedrich-Wilhelms-Universität Bonn

Endenicher Allee 60

53115 Bonn, Germany

E-mail: hensel@math.uni-bonn.de 